\title{
Effect of Clay Loading in the Morphology and Electrical Conductivity of Polyaniline-Clay/Elastomer Semiconductors
}

\author{
C. M. De León-Almazán ${ }^{1}$, I. A. Estrada-Moreno², J.L. Rivera-Armenta ${ }^{1}$, U. Páramo-García ${ }^{1}$ \\ 1. Centro de Investigación en Petroquímica Secundaria. Instituto Tecnológico de Ciudad Madero. Prol. \\ Bahía de Aldair y Av. de las Bahías, Parque de la pequeña y mediana industria, Altamira, Tamaulipas, \\ México. \\ 2. CONACyT - Centro de Investigación en Materiales Avanzados, S.C., Miguel de Cervantes 120, \\ Chihuahua, Chihuahua, 31136, México
}

Polyaniline (PAni) has ranked as one the most studied conducting polymers due to its outstanding combination of chemical nature, high electrical conductivity, environmental stability, harmlessness, ease of preparation and low cost. These attributes have positioned PAni in a wide range of applications such as rechargeable batteries, EMI shielding, microwave absorption, capacitors, electrochromic devices, electromechanical actuators, anticorrosion coatings and sensors[1]. Also, PAni has been considered as a viable alternative to replace metals as electrically conductive fillers in the production of semiconducting elastomers [2]. However, the main drawback of PAni lies in its lack of solubility and processability [3].

A nanoscaled conductive filler may easily form a conductive network even at low filler loadings. The confinement of PAni chains within the spaces of layered inorganic materials - like clays - is the most common strategy to prepare nanostructured PAnis. The preparation of PAni-clay nanocomposites (PCNs) is intended to improve both the affinity and electrical conductivity of PAni [2] to obtain a semiconducting elastomer suitable as a pressure sensor. Soto-Oviedo [4] and Sudha [2] pioneered at the study of PAniclay/elastomer (PCE) blends. However, since both of them follow a 1:1 clay:aniline ratio, the effect of low clay loads in the morphology and electrical behavior of PCE materials has not been evaluated so far.

PAni-clay/elastomer materials are prepared by a two-step procedure: first, the synthesis of PCNs by in situ oxidative polymerization of aniline in the presence of clay[1] modifying clay/aniline ratio, and then, the preparation of PCE by mechanical mixing of PCN with SEBS (Dynasol) employing a Brabender Intellitorque mixing chamber, keeping a 20:80 PCN:SEBS ratio. PCNs morphology was analyzed by using a JEOL JSM-7401F equipment. The electrical conductivity of PCNs and PCEs was measured with a HP Agilent Multimeter 34401 and a Keithley 6517A electrometer, respectively, by using a 4-probe device.

Figure 1.a-e shows the SEM micrographs of bare PAni and PCNs at 30,000x magnification. It is observed that bare PAni morphology (Figure 1.a) consists of 50-100 nm thick nanofibers, as already reported [1]. The presence of clay does not produce a significant effect on PAni morphology at any clay/aniline ratio (Figure 1.b-e) since PCNs keep the nanofiber structure trend. This result does not match with the one from Do Nascimento, who reported a shift to granular morphology with decreasing clay loading [5].

Figure 2 presents the electrical conductivity measurements of PCNs and PCEs. Opposite to the findings from Do Nascimento [5], it is observed that electrical conductivity of PCNs is increased along with clay/aniline ratio and the highest value is reached at 1:1 ratio, an unexpected result since 1:1 PNC has the highest amount of clay -a non-conducting filler-. On the contrary, the addition of clay produces a reduction in electrical conductivity of PCEs at 0.01 and 0.03 ratio, but it is slightly increased at 0.05 , which corresponds to the percolation threshold concentration. Notwithstanding, a dramatic decrease is 
observed at 1:1 ratio, as consequence of a lower PAni content in the PCE blend due to a higher amount of clay. This different behavior is not fully understood yet.

In conclusion, the variation of clay loading in the preparation of PCE blends does not affect PCNs morphology, but it does impact the electrical conductivity of both PCNs and PCEs in a significant way.

\section{References:}

[1] C.M. De León-Almazan, et al., Synth. Met. 236 (2018) 61-67.

[2] J.D. Sudha, et al., Compos. Sci. Technol. 69 (2009) 358-364.

[3] M. Jaymand, Prog. Polym. Sci. 38 (2013) 1287-1306.

[4] M.A. Soto-Oviedo, et al., Synth. Met. 156 (2006) 1249-1255.

[5] G.M. do Nascimento, et al., Polymer 47 (2006) 6131-6139.

[6] The authors thank Eng. Wilber Antunez Flores from CIMAV because of his kind help and Laboratorio Nacional de Nanotecnología, CIMAV.

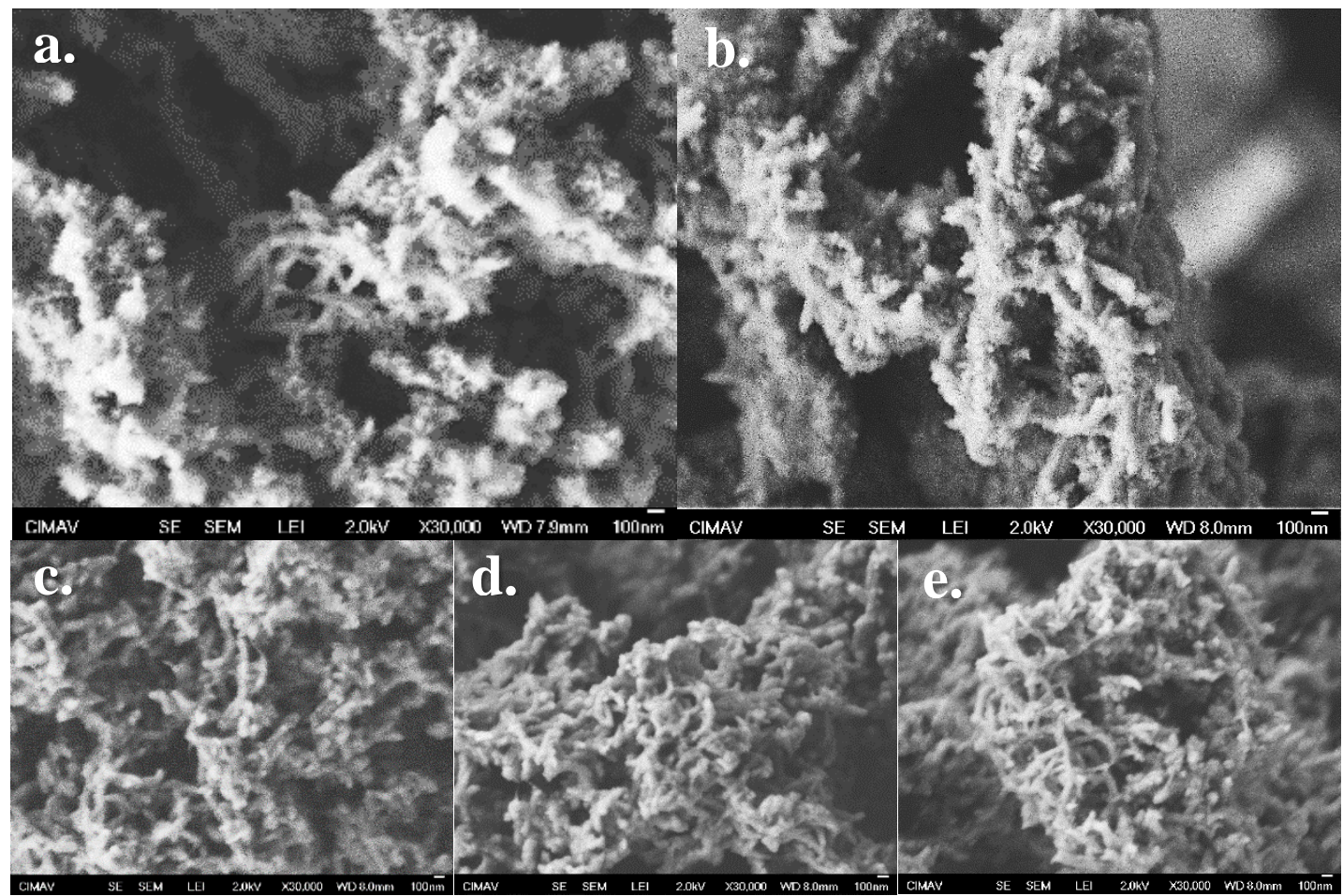

Figure 1. SEM micrographs of bare PAni (a) and PCNs with 1:1 (b), 0.01 (c), 0.03 (d) and 0.05 (d) clay/aniline ratio at $30,000 \mathrm{x}$
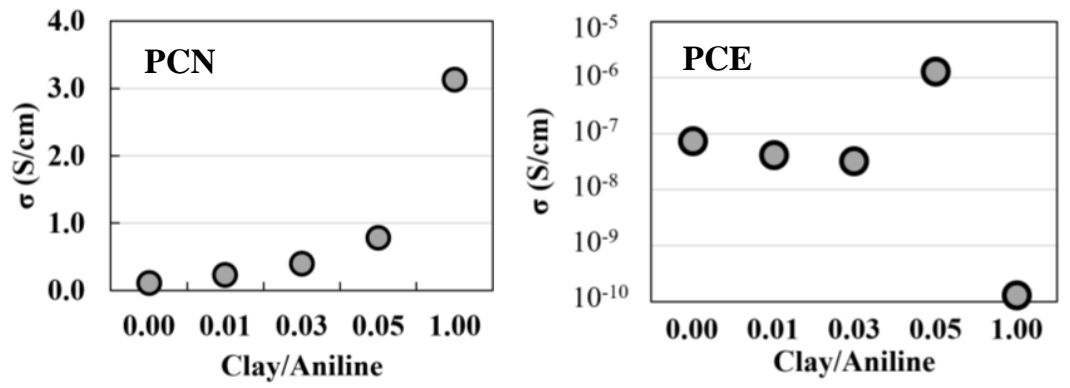

\begin{tabular}{c|cc}
$\begin{array}{c}\text { Clay/Aniline } \\
\text { ratio }\end{array}$ & \multicolumn{2}{|c}{$\boldsymbol{\sigma}(\mathrm{S} / \mathbf{c m})$} \\
\hline $\mathbf{0 . 0 0}$ & 0.1192 & $7.7082 \times 10^{-8}$ \\
$\mathbf{0 . 0 1}$ & 0.2366 & $4.2644 \times 10^{-8}$ \\
$\mathbf{0 . 0 3}$ & 0.4052 & $3.3625 \times 10^{-8}$ \\
$\mathbf{0 . 0 5}$ & 0.7825 & $1.3439 \times 10^{-6}$ \\
$\mathbf{1 . 0 0}$ & 3.1238 & $1.3261 \times 10^{-10}$
\end{tabular}

Figure 2. Electrical conductivity $(\mathrm{S} / \mathrm{cm})$ of PCNs and PCEs 